\title{
Jaw Cancer Detection Using Nano Amperometric Biosensor with Surface Tension Variables
}

\author{
K. Kalyan Babu \\ Department of Electronics and Communication Engineering, Gitam University, Visakhapatnam, AP, India
}

Received: February 07,2017; Accepted: April 05,2017; Published: April 20,2017

*Corresponding authors: K. Kalyan Babu, Assistant Professor, Department of Electronics and Communication Engineering, Gitam University, Visakhapatnam, AP, India, Tel: 08497917773;E-mail: kalyankillana@gmail.com

\begin{abstract}
Jaw cancer is detected by Nano amperometric biosensor with surface tension variables in this paper. Amperometric biosensor consists of three terminals namely Working Electrode, Reference Electrode and Counter Electrode. Jaw Cancer is highly dangerous disease seen in India. It is seen in people who smoke; eat Gutka, Khainas and excessive pans. Jaw cancer effects mouth and lungs also. In this paper JAW cancer symptoms, causes, eradication and prevention, drug delivery is discussed. Biosensor with Nano technology detects the jaw cancer with Glucose oxidase in this paper.
\end{abstract}

Keywords: Jaw Cancer; Biosensor

\section{Introduction}

Biosensors are invented by Micheal Clarke in 1962 They are of mostly electro chemical, these types of Biosensors are

1. Amperometric 2. Voltametric 3. Conductometric Biosensors [1]. In amperometric biosensor, current is final output, this current is measured in terms of oxygen. Whereas in Voltametric biosensor and voltage is final output. In conductometric biosensor, thermal voltage is final output. The biosensor contains a ligand and a transducer ligand means biological element and transducer mean which converts one form of energy to another form of energy the transducer output is an electrical signal measured either in volts or amperes. Biosensors are playing key roles in the field of Biomedical Engineering in detection of pathogens. Pathogens mean virus or fungi present in human body. The importance of Biosensors is increasing day by day in the field of Biomedical Engineering because of their accuracy, more sensitivity, less error ability.

When compared to other methods for detection of cancer like Biopsy, Biomarkers, they are very costly in medical grounds. But whereas Nano biosensor requires a thin film of Gold, Silver and platinum, which are very cheap when compared to present market rates. Thin films of micro depth in nature are taken and with the chemical method called Direct Current Cyclic Voltametry (DCCV) which is a direct indication of cancer in terms of oxygen in turn which is measured in terms of current.

This method is similar to photo synthesis in plants, where they inhale carbon dioxide and release oxygen. DCCV method exhales oxygen in Vitro and the release of oxygen is measured in terms of current (Figure 1).

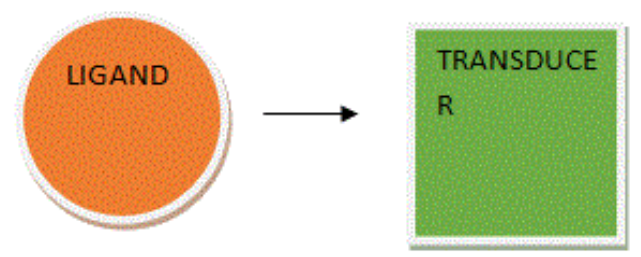

Figure 1: The above diagram shows the internal block diagram of a biosensor ligand means biological element and transducer means an instrument which converts a biological signal to an electrical signal.

\section{Construction}

Generally, Biosensors are constructed on glass substrates it is also called as Cell in technological terms. There are three colors which illustrate the internal structure of a biosensor [2]. Green color corresponds to working electrode, red color corresponds to reference electrode and blue color corresponds to counter electrode. The working electrode is made of glassy carbon, reference electrode is made of $\mathrm{Ag}, \mathrm{AgCl}$ and counter electrode is made of platinum

\section{Simple experimental procedure}

The working electrode is connected in Bare Glassy carbon and dipped in Ferro ferri solution and it is connected to Elecctro com software. If the output voltage is $0.01 \mathrm{~V}$ then the biosensor is made ready for detection of pathogenes. Next the biosensor is Pani tested which means Electro Chemical polymerization of Aniline.

A.Next the biosensor is dipped in GODx chemical. Glucose oxidase chemical is very costly and very rare to purchase. It takes minimum six months to purchase this chemical.

B.GODx chemical reacts with oxygen and produces Glucone Lactone and Hydrogen Peroxide at Anode.

C.Hydrogen Peroxide reduces to H20 and Oxygen gas.

D.Next I would like to intimate the meaning of Analyte.

E.Analyte means the analysis on which the test is performed.

F.This experiment, the analyte is blood sample. 
G.The detection is done by clicking Load Cell, the deviation in current is clearly observed.

H.If the current decreases, the blood sample analyte is having Cancer pathogen, otherwise no cancer.

The above procedure is repeated for all different cancers

\section{Nano Technology}

Nano Technology refers to 10 exp (-9). Nano materials are Graphene, Nanowires. Graphene is best suited for biological purposes [3]. Graphene Layer is embossed on top most layer of biosensor, for detection of cancer pathogen.

\section{Final Construction of Cell}

The amperometric biosensor which contains 3 electrodes as specified in to above paper rests on Glass Substrate in experimental procedure [4]. But in this simulation paper GaAs MESFETS are used as substrates. So I can use Semi Insulating GaAS substrate and on it the electrodes of Biosensor are resided (Figure 2).

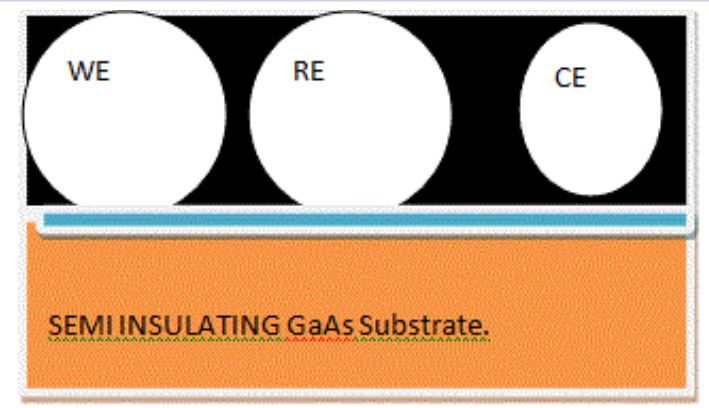

Figure 2: The above diagram illustrates the Simple diagram of Biosensor Cell gallium Arsenide substrate .Upon the substrate, the three terminals of Biosensor Cell are resided.

Above the substrate and below the terminals of Cell, lies the Graphene Layer, which is in Sky blue color.

\section{Symptoms of Jaw Cancer}

-Loss of teeth [5]

- Lose of taste on tongue

-Sour throat

-Loose Gums

-Lose of shape of Nose and Mouth

-Sudden and prolonged loss of skin in the jaw area

\section{Causes of Jaw cancer}

1.This mainly causes due to uneven cell division of living cells in the jaw area [5].

2.The Root cause of jaw cancer is because of undefined cell division of DNA which kills the healthy cells around mouth area and in jaws.

3.Another cause of Jaw Cancer is lack of vitamins.
4.The vitamin B deficiency leads to jaw cancer.

\section{Remedial Measures}

1.People should not get addicted to drinking, smoking and eating harmful pans.

2.People who have bad habits are advised to take the hot drink, smoking cigarettes in limited manner [5].

3.It is better to be away from harmful Khainas, Gutkas who are seen everywhere in India.

4.Especially in North India, almost all youth got addicted to the above said harmful foods by eating them in casual manner every day and every minute.

5.These harmful powders are made from dangerous drugs.

6.So I final conclude this paper by advising people like youth should not get addicted to above said consumables.

\section{Medicine delivery}

1.People who got jaw cancer are treated by doctors by Bio markers, Biopsy and radiation therapy [5].

2.Mostly the jaw cancer patients are treated by removing the cancer area through Surgery.

3.0nly $50 \%$ cases are successful, the remaining 50\% cases are failures.

4.Even after surgery, people are not completely curable; this results in death in many cases.

5.Generally the medicines are given through Swallowing; Injections etc. But in my research specifies the method of treatment through Nano Horns.

6.Nano Horns are best method of treating Cancer cases. Delivering medicine through Injection first mixes with blood and starts its action.

7.But by using Nano Horns, the medicine directly enters into Cancer zone and starts its functioning effectively rather than using Injections and Tablets.

\section{Surface Tension Variables}

1.Surface Tension is defined as cohesive forces between molecules of Liquid [6].

2.Examples: Mosquitoes walking on water, water beeding on Leaves and water leakage in tap in off condition.

3.Surface tension is denoted by $\mathrm{r}=\mathrm{F} / \mathrm{L}$. F stands for Cohesive force and L stands for Length of the assumed surface.

4.In surface tension, contact angles play a key role.

5.Contact angles can be obtrusive and acute on the assumed surface of the liquid.

\section{Core Theory of the Paper}

1. The core theory of the paper in my research is take the semi insulating GaAs substrate which works very quickly 
as it operates on Velocity of Light.

2.Insert the Graphene plate on the topmost surface of the Substrate [7].

3.Fix the three terminals of the Biosensor Cell.

4.Complete the experimental set up as discussed in the above part of the paper.

5.Pour the chemicals and Blood analyte into the Setup.

6.Connect the entire system to Electro com Software.

7.Now let us calculate the Variable r.

8.The cohesive force in the living cell is very high per unit length.

But whereas in Cancer cell, the cohesive force is very low per unit Length

\section{Governing Equations}

1. $\partial \mathrm{S} / \partial \mathrm{t}+\partial \mathrm{r} / \partial \mathrm{t} \rightarrow \partial \mathrm{P} / \partial \mathrm{t}[1]$

$S$ stands for substrate, $r$ represents Surface tension variable and $\mathrm{P}$ stands for product.

$\partial \mathrm{S} / \partial \mathrm{t}$ represents the rate of change of substrate concentration,

$\partial \mathrm{r} / \partial \mathrm{t}$ represents the rate of change of surface tension variable.

$\partial \mathrm{P} / \partial \mathrm{t}$ represents rate of change of product which is measured in terms of oxygen gas.

2. $\partial \mathrm{S} / \partial \mathrm{t}+\partial \mathrm{E} / \partial \mathrm{t}+\partial \mathrm{r} / \partial \mathrm{t} \rightarrow \partial \mathrm{P} / \partial \mathrm{t}[8]$

The above equation represents the Governing Equations with and without Enzymes.

\begin{tabular}{|c|c|c|c|c|}
\hline S & E & r & P & RESULT \\
\hline HIGH & NO & HIGH & HIGH & UNKNOWN \\
\hline LOW & NO & LOW & LOW & SUSPICIOUS OF CANCER \\
\hline HIGH & PRESENT & HIGH & HIGH & DEFINETLY NO CANCER \\
\hline LOW & PRESENT & HIGH & HIGH & NO CANCER \\
\hline
\end{tabular}

S = Substrate concentration

$\mathrm{E}=$ Enzyme concentration

$\mathrm{P}=$ Product concentration

\section{Usage in Home for Detection of Cancer}

My research configures to bring up a nano amperometric biosensor with 3 electrodes composed of Gold, Silver and Platinum calibrated in terms of current which is a direct measure of oxygen present in human body. It can be calibrated in terms of milli amperes for Non Cancer blood samples and order of nano amperes and pico amperes for cancerous blood samples [9].

My goal is to have nano amperometric biosensor in each and every home within next 5 years in early detection of any cancer before meeting a physician. It is similar to a thermometer which is used to detect fever in home and next going to a physician, with my biosensor we can confirm the presence of cancer pathogen at our home and then next meeting the physician for further treatment.

\section{Numerical Simulation}

Matlab program is written with the above variables and the results are tabulated.
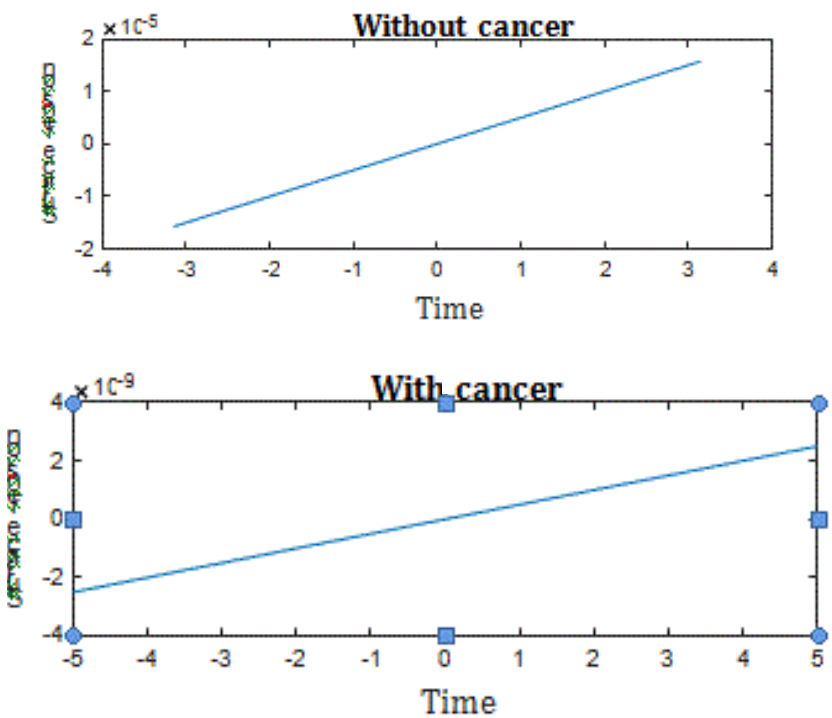

§The above two graphs illustrate the difference between the Non Cancer and Cancer outputs. The without cancer cell has very HIGH order of exp (-5), the cancerous cell will have LOW order of exp (-9). This paragraph illustrates the numerical simulation of the surface tension variable vs time. The without has high surface tension variable because the force acting on living healthy cell is very high (order of $10 \exp (-5))$ and the force on cancer cell is literally zero newtons (order of 10exp (-9)).This is proved numerically in Matlab program.

\section{Advantages of DCCV over Conventional Methods of Detecting Cancer}

1.This method of DCCV is more accurate than Biopsy, Biomarkers etc [10].

2.This method gives rise to formation of an instrument to detect cancer in house itself like a thermometer which is used to detect fever.

3.My future scope of work is to fabricate biosensor to have in each and every home which is used for detecting cancer at early stage and then meeting a physician [11].

\section{Conclusion}

In this paper, I have discussed the Cancer of Jaw being detected with Surface tension variables in Simulation. I have also referred to Medicine delivery through Nano horns and remedial measures. So, I finally conclude that Prevention is better than Cure. It is always better to be away from smoking and drinking. This method is comparatively better than conventional methods like Biomarkers, Biopsy tests for detection of cancer. They are very costly whereas this method requires a small blood sample to 

Variables

detect the cancer at initial stage.

\section{Acknowledgements}

I would thank my Ph.D guide Professor Prof. D. V. Rama Koti Reddy HoD, Dept. of Instrument Technology, for his continuous support and Encouragement.

\section{References}

1. Romas Baronas, Ivanauskas Feliksas, Juozas Kulys. Modeling biosensors at steady state and internal diffusion limitations. Mathematical Modeling of Biosensors. 2010;9:9-20. doi: 10.1007/97890-481-3243-0_2

2. Winnie WY Chow, Yanli Qu, Wen J Li. Carbon-Nanotube-Based Fluidic Shear-Stress Sensors. 2010;31-68. doi: 10.1201/b10450-3

3. Andre P. Garcia, Dipanjan Sen, Markus J. Buehler. Nature's Flexible and Tough Amour: Geometric and Size Effects on Diatom-Inspired Nanoscale Glass. 2011;273-304. doi: 10.1201/b11384-11

4. Lianxi Zheng, B.C. Satishkumar. Carbon-Nanotube-Based Sensors. 2010;1-30. doi: 10.1201/b10450-2

5. WIKIPEDIA- JAW CANCER

6. Wikipedia-Surface Tension

7. Romas Baronas, Ivanauskas Feliksas, Juozas Kulys. Modeling biosensors at steady state and external diffusion limitations. Mathematical Modeling of Biosensors. 2010;9:21-26. doi: 10.1007/978-90-4813243-0_3

8. Romas Baronas, Ivanauskas Feliksas, Juozas Kulys. Modeling non stationary state of biosensors. Mathematical Modeling of Biosensors. 2010;9:33-39. doi: 10.1007/978-90-481-3243-0_5

9. Kilho Eom, Taeyun Kwon. Nanomechanical in Vitro Molecular Recognitions: Mechanical Resonance-Based Detections. 2011;331370. doi: 10.1201/b11384-13

10.Romas Baronas, Ivanauskas Feliksas, Juozas Kulys. Modeling biosensors utilizing microbial cells. Mathematical Modeling of Biosensors. 2010;9:27-31. doi: 10.1007/978-90-481-3243-0_4

11. Shoumen Palit, Austin Datta. Future Healthcare: Bioinformatics, NanoSensors, and Emerging Innovations. 2010;247-312. doi: 10.1201/ b10450-9 